\title{
Soil parameters deduced by refraction microtremor method
}

\author{
Wenhui Zhang, Weijun Jin, Weihang Huang \\ Institute of Geology and Geophysics, Chinese Academy of \\ Science, Beijing, China
}

\author{
Rui Cui \\ Schlumberger Technology Services (Beijing) Ltd, Beijing, \\ China
}

\begin{abstract}
Soil parameters have important impacts on the response to earthquake damage. Refraction microtremor (REMI) method is of practical significance to determine the soil thickness and velocity structure of the site. The REMI method acquires data by the conventional refraction seismic equipment, which makes use of ambient seismic field as the source. Transformation from the time-space domain to the slowness - frequency domain will be carried out in the REMI method to extract the dispersion curve by means of the tau-p transformation and the Fourier transformation. Different from the surface wave exploration of active source, the soil structure will be achieved in the greater depth by the dispersion curves inversion.

Refraction microtremor method uses the conventional seismic recording instruments during the progress of seismic data acquisition. It takes about $40 \mathrm{~s}$ in one acquisition, which is a relatively short cycle time. This is an available and high efficiency way to collect seismic data. REMI method extracted dispersion curve from P-F domain. Because the linear slowness is corresponding to the nonlinear velocity of surface wave, the change of velocity is much more obvious. As a result, it is easier to distinguish primary order and high order dispersion curves on the energy spectrum diagram. Soil structure can be obtained from the inversion progress. Because of natural vibration wave field, such as cars, trains and planes, REMI method has lower frequency characteristics, which makes REMI method having a greater exploration depth, generally reaching above 100 m.Keywords-Transient electromagnetic method; Threedimensional forward modeling; Direct time-domain by vector finite element mothed; Ramp time
\end{abstract}

Keywords-REMI; soil parameters; tau-p transformation; slowness - frequency domain

\section{INTRODUCTION}

In this research, we gathered the seismic data in open-pit mine located in Inner Mongolia. Then we calculated the relationship between surface wave velocity and frequency. Dispersion curves were picked out one by one from the images of velocity and frequency. After that, we build a simple initial velocity model according to the geology settings. At last, we obtained the curves of phase velocity and depth. From these curves, the structures of stratum underground are presented clearly. By comparing the curves to the drill bore data, we found good consistency between them. In other words, using REMI to study the structure underground is effective and economic.

During the data acquisition process, active source seismic exploration has some inevitable difficulties. For example, mechanical fall source could not supply enough energy and explosive source are not allowed in city due to the security issues. Thus the precision, depth and applicability of seismic exploration are limited to some extent. Equipment and the layout method of refraction microtremor are the same with refraction exploration.

Repeated tests in different places showed that refraction microtremor method is an economic and convenient way to get the shear wave velocity model of high accuracy up to $100 \mathrm{~m}$ underground. What's more, 2D array can obtain the velocity model up to $1000 \mathrm{~m}$ underground.

\section{THEORY OF REMI}

The $\tau$-p transform is the first step of REMI research. Thorson and Claerbout have achieved important progress in REMI. Seismogram amplitudes of time and distance (x-t) are transformed to amplitudes of ray parameter and intercept time $(\tau-p)$. This is the first step in P-wave refraction velocity analysis which was used by Clayton and McMechan in 1981. Rayleigh wave phases are separated from other arrivals in dispersion curve of $\mathrm{p}-\mathrm{f}$ domain. The P-f technique was first used by Park to active source MASW records in 1998. From the curves of dispersive phases, it is obvious that curves are sloping down from low slowness (p) at low frequencies to high slowness at higher frequencies.

$$
S(\mathrm{p}, \tau)=\int_{x} \varphi(x, t) d x
$$

To be discrete, it could be written as:

$$
S(p, \tau)=\sum_{j=0}^{n x-1} \varphi(x, t)
$$

It is important but not easy to pick and interpret dispersion curves directly from the $\mathrm{p}-\mathrm{f}$ images. Spectral power-ratio calculation is effective in dispersion picking. Picking phase velocities at the frequencies where a slope or a peak in spectral ratio occurs clearly locates the dispersion curve. Picks are not made at frequencies without a definite peak in spectral ratio, often below $4 \mathrm{~Hz}$ and above $14 \mathrm{~Hz}$ where an identifiable dispersive surface wave does not appear. Spectral power-ratio is computed in this way:

$$
R(|p|, f)=S(|p|, f) n p /\left[\sum_{l=0}^{n p-1} S(|p|=l d p, f)\right]
$$

Usually, average velocity of 30 meters underground could be presented in $p-f$ image. Because of the simple liner array, 
apparent velocity will be larger than the real velocity when the seismic wave arrived in some incidence.

$$
V_{a}=V / \cos (a)=1 / p
$$

So in the interpretation of data can not only choose the phase velocity in single frequency. In order to avoid this problem, you need to find the minimum speed rate of spectrum peaks place to make an envelope.

Similar to other geophysical inversion problems, inversion of refraction microtremor dispersion curves can be solved by using the least square method. But because of the complexity between the strata model and surface wave velocity, the precision of the initial model is crucial. This initial model can only be obtained by experience in practice. After a lot of research work, it is more commonly to use nonlinear optimization method. For example, as a global algorithm, genetic algorithm there is not too much importance on initial model. More over, its result is much more stable and reliable. At the same time, it needs more CPU time to achieve the result.

\section{EXAMPLE}

In this research, we collected the seismic data in a mine located in Inner Mongolia. A 24-channel array of $10 \mathrm{~Hz}$ geophones $500 \mathrm{~m}$ long was laid out along the side slope of the mine. $8-12 \mathrm{~Hz}$ exploration geophones can give surface wave phase velocities at frequencies as low as $2 \mathrm{~Hz}$ and as high as $20 \mathrm{~Hz}$. This range is appropriate for constraining shear velocity profiles from the surface to $150 \mathrm{~m}$ depth. The microtremor technique may be most fruitful where noise is most severe. Fig 1-3 show the curve of dispersion, the inversion result of the velocity model and the velocity profile.

Fig.1 shows the velocity spectrum and corresponding dispersion curve and picks. Then the picks are modeled to produce the shear wave velocity model as Fig. 2 shown. The site is full of noise generated from loading and unloading of containers from heavy trucks inside the mine site. Fig.3 is one of the profiles of velocity in our research. There is a low velocity level at the surface of ground. This level is called weathered layer. Under this level, there is a higher velocity level. This is where mine level located in. At the depth of about $40 \mathrm{~m}$, there is a clear interface. It could be explained as the bottom of the mine level. This result could match the $10 \mathrm{~m}$ thickness of the mine layer in drill data showed in Fig.4.

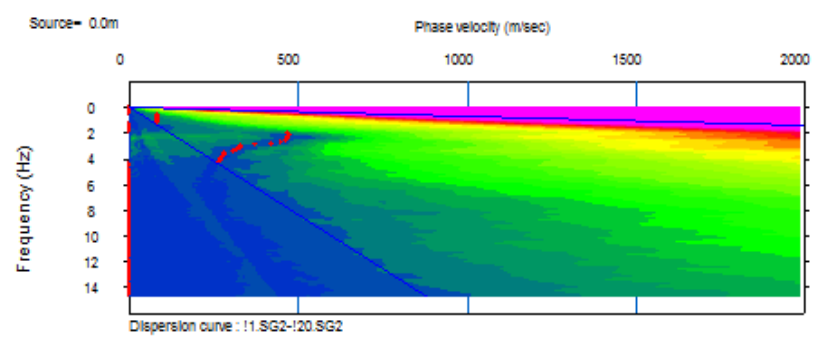

Fig.1 Dispersion curve (marked in red dots )

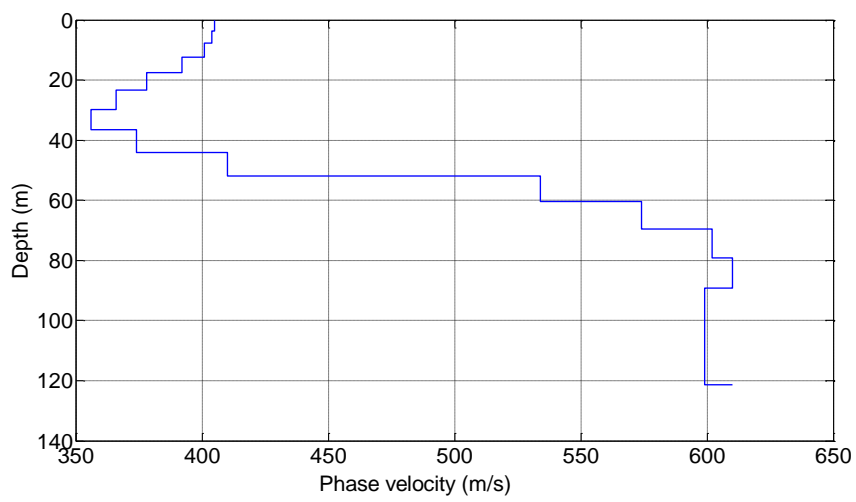

Fig.2 Velocity structure from inversion

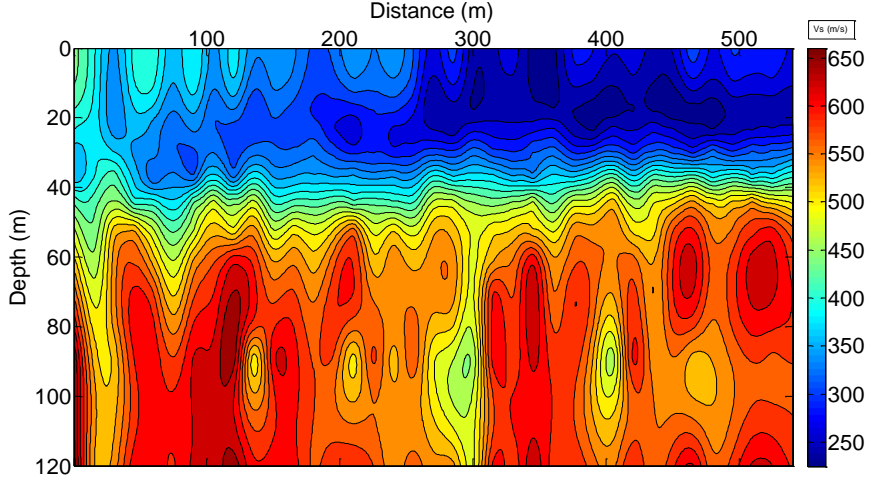

Fig.3 Profile of velocity

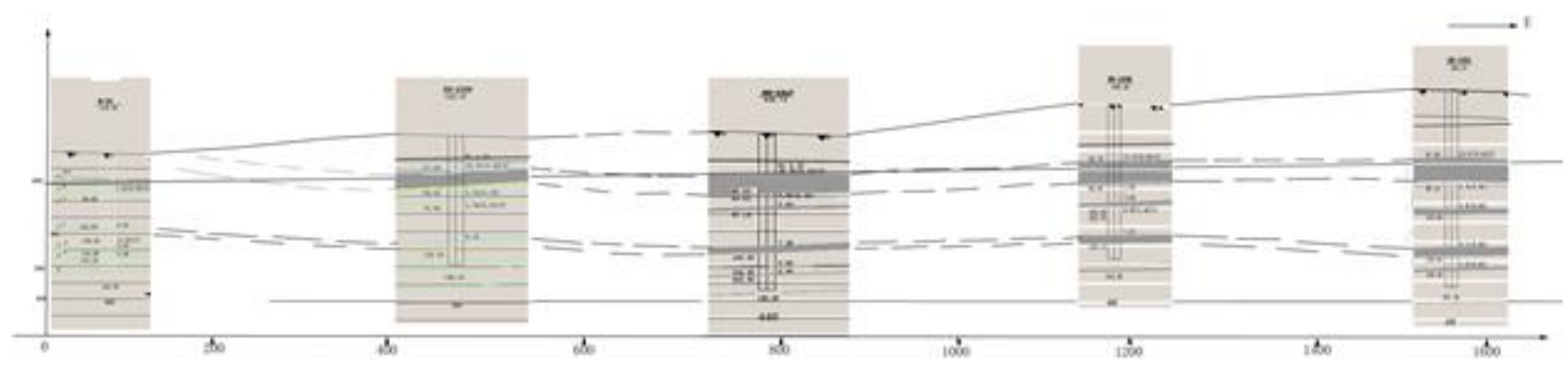

Fig.4 Positions of drill holes and depth of mine underground (Gray layer in drill) 


\section{CONCLUSIONS}

Refraction microtremor is much more convenient and economic than other microtremor methods. Human living places are located in modern basin environment, which is a typical dual structure. Characteristics of the refraction wave in the progation of rock and soil are different. As a result, REMI method is feasible to obtain velocity structure and other parameters in shallow surface.

\section{REFERENCES}

[1] Mohamed Ahmed Gamal, Satish Pullammanappallil, 2011, Validity of the Refraction Microtremors (ReMi) Method for Determining Shear Wave Velocities for Different Soil Types in Egypt: International Journal of Geosciences, 2, 530-540 